\title{
A Compilation and Meta-analysis of Salmon Diet Data in the North Pacific Ocean
}

\author{
Caroline Graham ${ }^{1,2}$, Evgeny A. Pakhomov ${ }^{1,2}$, and Brian P.V. Hunt ${ }^{1,2,3}$ \\ ${ }^{1}$ Institute for Oceans and Fisheries, University of British Columbia, 2202 Main Mall, Vancouver, British Columbia \\ V6T 1Z4, Canada \\ ${ }^{2}$ Department of Earth, Ocean, and Atmospheric Sciences, University of British Columbia, 2207 Main Mall, \\ Vancouver, British Columbia V6T 1Z4, Canada
}

${ }^{3}$ Hakai Institute, PO Box 25039, Campbell River, British Columbia V9W 0B7, Canada

Keywords: salmon, diet, meta-analysis, trophic niche

Although the freshwater phase of the salmon life cycle has been studied in depth, there is much less information available on the marine phase, even though Pacific salmon can spend anywhere from one to seven years of their life in the ocean. With rapidly changing ocean conditions, it is important to understand this phase of the salmon life cycle. One of the most significant factors affecting the survival of salmon is the presence and abundance of nutritious prey. Although it is difficult to measure prey occurrence across the scale of the Pacific Ocean basin, information on prey presence and abundance can be obtained by studying salmon diets. Diet data can give insight into food webs, niche overlap between species/stocks, potential competition, health, and changing ocean conditions. Over the past century, there has been sporadic research on salmon diets in the ocean, and inconsistent methods have been used to quantify this information. There is an urgent need to consolidate available data in a useful way to understand salmon habitat, identify knowledge gaps, and project future changes.

In order to address this issue, we conducted a systematic review of the literature, including a keyword search using several online databases (Proquest Aquatic Sciences and Fisheries Abstracts, Web of Science Core Collection and Web of Science Zoological Record) and supplemented this with literature from the North Pacific Anadromous Fish Commission's and International North Pacific Fisheries Commission's documents and bulletins. We identified over 250 relevant sources, containing marine salmon diet data — specifically in the form of stomach content information. Diet data was found for Chinook, coho, sockeye, chum, and pink salmon, as well as steelhead, across the North Pacific basin. These data will be extracted and collated into an open-access diet database to help address questions related to salmon marine survival and will also represent a valuable resource for modeling efforts aimed at Ecosystem Based Management and understanding North Pacific ecosystem response to climate change.

Acknowledgements - We would like to acknowledge Marina Espinasse for her hard work on compiling the initial database. Thank you to the Ambrose Monell Foundation and the G. Unger Foundation for providing funding. Also, thank you to the Pelagic Ecosystems Lab and the University of British Columbia for supporting this research project. 